\title{
Treatment failure in tuberculosis
}

\author{
J.R. Panickar and W. Hoskyns
}

ABSTRACT: Treatment of latent tuberculosis (TB) infection with 3 months of rifampicin/isoniazid is a major part of preventive TB programmes. The effectiveness of treatment of latent TB infection can only be assessed by rates of subsequent breakdown and there are few outcome data for this combination of rifampicin/isoniazid. Therefore, the aim of the present study was to estimate the failure rate following treatment for the latent TB infection.

A questionnaire survey was carried out in all parents of children aged $<\mathbf{1 6}$ yrs who completed treatment for latent TB infection at Leicester Royal Infirmary (Leicester, UK) over the period 19972003. Cases of treatment failure were identified by reviewing all re-referrals to the clinic, identifying children developing TB while on treatment and by postal questionnaire to all patients discharged.

Of the 400 eligible children, 344 (86\%) replied. Three children who had latent TB infection subsequently developed TB disease over the time period. Of those three patients, one developed chest radiograph signs at the end of treatment and two presented with symptoms within 2 yrs of completing treatment. Overall, the mean treatment failure rate was $0.87 \%(95 \%$ confidence interval $0.3-2.5)$ or 2.2 cases per 1,000 patient-yrs.

In conclusion, rates of tuberculosis breakdown after treatment for latent tuberculosis infection with 3 months rifampicin/isoniazid are acceptably low.

\section{KEYWORDS: Latent tuberculosis infection, treatment failure}

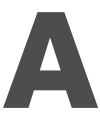

fter many years of decline, the rates of tuberculosis (TB) incidence in the UK have remained static and even increased [1]. One of the difficulties in the control of the spread of $\mathrm{TB}$ is that, once infected, the disease may remain dormant for many years. Strategies for the prevention of TB consist firstly of identification and treatment of TB disease and secondly of contact tracing and treatment of early disease and latent infection. Treatment of latent TB infection is designed to reduce the burden of future $\mathrm{TB}$ and this is particularly appropriate for young patients who have a higher risk of progression of disease.

There is data on the effectiveness of treatment of $\mathrm{TB}$ in adults [2-5] and it is assumed that treatment of children is probably similar, although specific data is scarce. There is less data on treatment of latent infections. In the UK, standard guidelines are available for the management of the TB disease and the standard treatment for latent TB infection is a 3-month course of rifampicin and isoniazid [6]. There is data on safety of this regime [7] but not on efficacy. The purpose of the present study was to estimate the failure rate following treatment of latent infection.

\section{METHODS}

\section{Population}

The study population was taken from the Leicester TB clinic (Leicester, UK) where all children on anti-TB treatment in the geographical area up to $16 \mathrm{yrs}$ of age are seen. The population is relatively stable and this makes the sample essentially population based.

\section{Data collection}

All children who completed treatment for latent TB infection at Leicester Royal Infirmary from January 1997 to December 2003 were included. The list of children was obtained from the clinic database, along with details of age, sex, bacille Calmette-Guerin (BCG) status, country of origin, source of referral and the relationship to any known index case. Cases of treatment failure were identified by the following three methods: 1) review of all re-referrals to the clinic; 2) identification of all children upgraded from latent infection to disease while on treatment; and 3) postal questionnaire to all patients discharged after completion of treatment. The questionnaire was initially piloted on 20 children attending the clinic to assess comprehensibility. All cases that had a second course of treatment were categorised by questionnaire and notes
AFFILIATIONS

Dept of Child Health, Leicester Royal Infirmary, Leicester, UK.

\section{CORRESPONDENCE}

W. Hoskyns

Dept of Child Health

Leicester Royal Infirmary

Leicester

LE1 5WW

UK

Fax: 441162587637

E-mail: wren.hoskyns@uhl-tr.nhs.uk

Received:

July 122006

Accepted after revision:

November 172006

STATEMENT OF INTEREST

None declared. 
review, either as having had further exposure to TB or as treatment failure. There was a repeat mailing for nonresponders after 1 month and remaining nonresponders were contacted by telephone after 2 months.

The questionnaire (Appendix 1) covered whether the child had had TB diagnosed since discharge and also asked about present symptoms, which might represent undiagnosed $\mathrm{TB}$, i.e. fever, cough, weight loss, night sweats or shortness of breath for $>3$ weeks. Responders with positive symptoms were offered a review appointment and repeat chest radiograph.

\section{Diagnostic pathway}

Referrals to the TB clinic came either from general practitioners, school screening or referrals from the TB contact tracing service. Children with symptoms were assessed and investigated for the presence of $\mathrm{TB}$ as appropriate. For asymptomatic contacts the TB nurses assessed the proximity of the contact to the index case and decided on the need for screening by a standard protocol. In the presence of significant contact, defined as $6-8 \mathrm{~h} \cdot$ week $^{-1}$ of close proximity in the home, work or school environment, all children $<5$ yrs of age were referred to the clinic. Children $>5 \mathrm{yrs}$ of age were initially screened and referred to the children's TB clinic if the Heaf test was positive (grade $2-4$ ) regardless of previous BCG vaccination. Screening for TB was performed in the clinic using a chest radiograph and Mantoux test (Evans Medical, Leatherhead, UK). Either one tuberculin unit (TU; with prior BCG) or 10 TU (no previous BCG) was administered intradermally on the left forearm and the test was read 48$72 \mathrm{~h}$ later. Induration of $\geqslant 5 \mathrm{~mm}$ was treated as a positive response. This assessment for latent $\mathrm{TB}$ infection in this population has been shown to be more restrictive than current British Thoracic Society guidelines but with little increase in risk for those not treated [8].

Asymptomatic Mantoux positive patients with a normal chest radiograph were regarded as having a latent $\mathrm{TB}$ infection and treated with a 3-month course of rifampicin and isoniazid (10 $\mathrm{mg} \cdot \mathrm{kg}^{-1} \cdot \mathrm{day}^{-1}$ and $5 \mathrm{mg} \cdot \mathrm{kg}^{-1} \cdot \mathrm{day}^{-1}$, respectively, rounded up to a convenient dose) [9]. Children were routinely reviewed in the clinic at 1 month and again at the end of treatment with additional appointments if clinically warranted. In addition, all patients were monitored in the community by the TB nursing service. On successful completion of a course of treatment, patients were discharged from the clinic.

\section{RESULTS}

In total, 400 children (228 males, 172 females) were treated for latent TB infection. The response rate was 50\% after the first questionnaire, $65 \%$ after repeat mailing and $85 \%$ after telephone contact. In total, 86\% (344 out of 400) responded and there was no difference between responders and nonresponders for baseline characteristics except for the increased age of the nonresponders.

The demographics of the children are given in table 1 . The majority of the children were born in the UK, had BCG vaccination and were referred following contact with a TB case.

In total, three children subsequently developed TB disease over the 5-yr period. Of these three patients, one developed chest radiograph signs at the end of treatment and in

\begin{tabular}{lc} 
TABLE 1 & \\
\hline Variables & \\
\hline & \\
Age yrs & 8 \\
Male sex & $228(57)$ \\
BCG & \\
$\quad$ Yes & $323(81)$ \\
No & $53(13)$ \\
Country of origin UK & $296(74)$ \\
Referral category & \\
$\quad$ Contact & $351(88)$ \\
Symptomatic & $7(2)$ \\
Others & $42(10)$
\end{tabular}

Data are presented as mean or $\mathrm{n}(\%)$. BCG: bacilli Calmette-Guerin.

retrospect was noncompliant with treatment. The treatment course was extended and the patient upgraded to a diagnosis of primary TB. The other two children were referred back to the paediatric TB clinic with symptoms within 2 yrs of completing treatment where concordance with treatment was felt to have been good. In total, three children (mean $0.87 \%$, $95 \%$ confidence interval $0.3-2.5 \%$ ) had treatment failure. This equates to 2.2 cases per 1,000 patient-yrs (table 2 ).

In total, 20 children had persistent symptoms in response to the questionnaire. They were contacted by telephone after 2 months. In total, 11 children who still had symptoms were clinically assessed and chest radiographs taken, but none were found to have TB. There were 12 children referred back to the clinic with a new contact with TB; however, none were found to have evidence of TB disease but this group is difficult to assess for presence of a newly acquired infection.

Complications in the present study were uncommon. The only child receiving pyrazinamide had drug sensitisation (the source case had isoniazid resistant TB) and the drug had to be substituted after 3 weeks because of a skin reaction.

\begin{tabular}{lc} 
TABLE 2 Results & \\
\hline $\begin{array}{l}\text { Children sent questionnaires } \\
\text { Questionnaire responders }\end{array}$ & 400 \\
$\begin{array}{l}\text { Follow-up time from completion of } \\
\text { treatment yrs }\end{array}$ & $344(86)$ \\
$\begin{array}{l}\text { Repeat referral following new TB } \\
\text { contact }\end{array}$ & 4.02 \\
$\begin{array}{l}\text { Upgraded during treatment } \\
\text { Re-referral no new contact } \\
\text { Identified from questionnaire }\end{array}$ & 12 \\
$\begin{array}{l}\text { Overall failure of responders } \\
\text { Rate of failure 1000 patient-yrs }\end{array}$ & 1 \\
\end{tabular}

Data are presented as $n, n(\%)$, mean $(95 \%$ confidence interval $(\mathrm{Cl}))$ or $\mathrm{n}, \%$ (95\% Cl). TB: tuberculosis. 


\section{DISCUSSION}

The present study has demonstrated a failure rate for the treatment of latent TB infection of $0.87 \%$ (2.2 per 1,000 patientyrs). The confidence interval is wide given the small number of treatment failures.

The response rate to the questionnaire was high and as the study was population based, the results of this study are probably applicable to other populations. The characteristics of the nonresponders were very similar to the responders except for the higher mean age. This presumably reflects the fact that adolescents and young adults are a more mobile population and more likely to have left the parental home. As this age group has a higher breakdown rate of TB [10] it is possible that the failure rate has been underestimated.

The questionnaire was simple and was pre-tested for comprehensibility. It also included questions about current symptoms and all patients who responded positively were reviewed in the clinic. No unrecognised TB was diagnosed by the questionnaire.

It is difficult to be precise about what the risk of progression from latent TB infection to TB disease in this group would be had they not been treated. They certainly represent a high-risk group, in that the majority had been in contact with infectious TB $(88 \%)$ and most of the school-age children had a positive Heaf test (grade 2 or more) prior to referral. A negative Mantoux test (1 TU) was used to try to reduce the known falsepositive effect of BCG before treatment was initiated [8]. The estimated lifetime risk of disease progression for a newly infected young child is $10 \%$ with roughly half of the risk occurring in the first 2 yrs after infection [10, 11]. This risk decreases with age to $\sim 1 \%$ at 20 yrs of age. The mean age of the present group was 8 yrs and $82 \%$ were followed for $>2$ yrs.

Most of the evidence for effectiveness of latent TB infection treatment has been with isoniazid monotherapy and was reviewed in 1970 by FEREBEE [12]. The populations recruited for these studies were varied and some included children with primary $\mathrm{TB}$ as, at the time of enrolment, uncomplicated primary TB was not routinely treated. The difficult diagnostic distinction between primary and latent TB persists and several studies have demonstrated that a chest radiograph has relatively low sensitivity in diagnosing enlarged hilar and mediastinal lymph nodes compared with computed tomography scan $[13,14]$. However, in the subgroup most similar to this study (namely, $<15$ yr olds in household contact with TB, tuberculin reactors or convertors, normal chest radiograph and followed up for $\leqslant 10 \mathrm{yrs}$ ) the rate of subsequent pulmonary or extra-pulmonary TB was 16.7 per 1,000 patients untreated and 5.7 per 1,000 post-treatment compared with the present figure of $0.87 \%$ (8.7 per 1,000$)$. However, methodological differences make comparisons difficult. In a small double blinded study of isoniazid versus placebo in young adult contacts of $\mathrm{TB}$, the rates of TB over 7 yrs for placebo and isoniazid treatment were 9.4 and $0.8 \%$, respectively [15]. Overall protection provided by isoniazid monotherapy for these and similar studies varied $70-95 \%$ and the failure rates on treatment are broadly in agreement with the present results.

A recent meta-analysis in adults compared 3-month dual isoniazid/rifampicin therapy against 6-12-month isoniazid monotherapy [16]. The overall results showed a treatment failure rate of 4.2 and $4.1 \%$, respectively. While this overall result supports the efficacy of combination treatment, the individual studies varied considerably in methodology and patient characteristics (e.g. HIV status and reason for TB testing) and none was specifically paediatric.

Alternative combinations of drugs have been used for latent TB infection treatment but have been less than satisfactory. Studies in adults using the rifampicin/pyrazinamide combination for 2 months have concluded that the risk of side effects is high [17]. In the present study, only one patient received this combination and reacted to the pyrazinamide component. The dosage of drugs used in this study was within the recommended therapeutic range $\left(5 \mathrm{mg} \cdot \mathrm{kg}^{-1}\right.$ isoniazid, $10 \mathrm{mg} \cdot \mathrm{kg}^{-1}$ rifampicin rounded up to the nearest convenient dosage), but there is recent evidence that this may give variable but generally low serum levels of isoniazid in children [18]. The significance of this finding is not clear for efficacy of treatment but may mean that higher doses of isoniazid monotherapy for latent TB infection would increase cure rates.

The present data shows that 3-month dual therapy with isoniazid/rifampicin has acceptably low failure rates and low side effects in this group of patients. Compliance with medication, as monitored in clinic and by the tuberculosis nursing service, seemed to be high but this was not assessed directly. Likewise, the potential economic savings of the reduced time of treatment monitoring compared with isoniazid monotherapy have not been estimated.

\section{APPENDIX 1}

\section{QUESTIONNAIRE}

Dear Parent/Guardian,

Your child was treated at the LRI on

1. Since discharge on has your child had any further problems with TB?

YES NO

If yes which hospital was your child treated in?

Approximate date treatment started:

Approximate date treatment finished:

2. Do you have any of the following TB symptoms now? (Please circle)

Fever for more than 3 weeks

Yes No

Cough for more than 3 weeks

Yes No

Weight loss for more than 3 weeks

Yes No

Night sweats for more than 3 weeks

Yes No

Shortness of breath for more than 3 weeks Yes No

Please give your present home/mobile telephone number in case we need to clarify any of the above details. 


\section{REFERENCES}

1 Control and prevention of tuberculosis in the United Kingdom: code of practice 2000. Joint Tuberculosis Committee of the British Thoracic Society. Thorax 2000; 55: 887-901.

2 A controlled trial of six months chemotherapy in pulmonary tuberculosis. First Report: results during chemotherapy. British Thoracic Association. Br J Dis Chest 1981; 75: 141-153.

3 Clinical trial of six-month and four-month regimens of chemotherapy in the treatment of pulmonary tuberculosis: the results up to 30 months. Tubercle 1981; 62: 95-102.

4 Controlled clinical trial of 4 short-course regimens of chemotherapy (three 6-month and one 8-month) for pulmonary tuberculosis. Tubercle 1983; 64: 153-166.

5 A controlled trial of 6 months' chemotherapy in pulmonary tuberculosis. Final report: results during the 36 months after the end of chemotherapy and beyond. British Thoracic Society. Br J Dis Chest 1984; 78: 330-336.

6 Chemotherapy and management of tuberculosis in the United Kingdom: recommendations 1998. Joint Tuberculosis Committee of the British Thoracic Society. Thorax 1998; 53: 536-548.

7 Ormerod LP. Rifampicin and isoniazid prophylactic chemotherapy for tuberculosis. Arch Dis Child 1998; 78: 169-171.

8 Hoskyns EW, Simpson H, Monk P. Use of the 1 tuberculin unit (TU) Mantoux test in the assessment of tuberculous infection in children following neonatal BCG vaccination. Thorax 1994; 49: 1006-1009.

9 British National Formulary for Children. Joint Formulary Committee. Infections. London, British Medical Association, 2006; pp. 280-396.
10 Comstock GW, Livesay VT, Woolpert SF. The prognosis of a positive tuberculin reaction in childhood and adolescence. Am J Epidemiol 1974; 99: 131-138.

11 Sutherland I. Recent studies in the epidemiology of tuberculosis, based on the risk of being infected with tubercle bacilli. Adv Tuberc Res 1976; 19: 1-63.

12 Ferebee $\mathrm{SH}$. Controlled chemoprophylaxis trials in tuberculosis. A general review. Adv Tuberc Res 1970; 17: 28-106.

13 Delacourt C, Mani TM, Bonnerot V, et al. Computed tomography with normal chest radiograph in tuberculous infection. Arch Dis Child 1993; 69: 430-432.

14 Swingler GH, duToit G, Andronikou S, van der Merwe L, Zar HJ. Diagnostic accuracy of chest radiography in detecting mediastinal lymphadenopathy in suspected pulmonary tuberculosis. Arch Dis Child 2005; 90: 1153-1156.

15 Veening GJ. Long term isoniazid prophylaxis. Controlled trial on INH prophylaxis after recent tuberculin conversion in young adults. Bull Int Union Tuberc 1968; 41: 169-171.

16 Ena J, Valls V. Short-course therapy with rifampicin plus isoniazid, compared with standard therapy with isoniazid, for latent tuberculosis infection: a meta-analysis. Clin Infect Dis 2005; 40: 670-676.

17 Lee AM, Mennone JZ, Jones RC, Paul WS. Risk factors for hepatotoxicity associated with rifampicin and pyrazinamide for the treatment of latent tuberculosis infection: experience from three public health tuberculosis clinics. Int J Tuberc Lung Dis 2002; 6: 995-1000.

18 Schaaf HS, Parkin DP, Seifart HI, et al. Isoniazid pharmacokinetics in children treated for respiratory tuberculosis. Arch Dis Child 2005; 90: 614-618. 